\title{
What Does Reduced FDG Uptake Mean in High-Grade Gliomas?
}

\author{
Caroline Bund, MD, *†+ Benoît Lhermitte, MD, $\S$ A. Ercument Cicek, PhD,// Elisa Ruhland, MSc, *† \\ François Proust, MD, ** and Izzie Jacques Namer, MD, PhD*广t
}

\begin{abstract}
Purpose: As well as in many others cancers, FDG uptake is correlated with the degree of malignancy in gliomas, that is, commonly high FDG uptake in high-grade gliomas. However, in clinical practice, it is not uncommon to observe high-grade gliomas with low FDG uptake. Our aim was to explore the tumor metabolism in 2 populations of high-grade gliomas presenting high or low FDG uptake.

Methods: High-resolution magic-angle spinning nuclear magnetic resonance spectroscopy was realized on tissue samples from 7 high-grade glioma patients with high FDG uptake and 5 high-grade glioma patients with low FDG uptake. Tumor metabolomics was evaluated from 42 quantified metabolites and compared by network analysis.

Results: Whether originating from astrocytes or oligodendrocytes, the highgrade gliomas with low FDG avidity represent a subgroup of high-grade gliomas presenting common characteristics: low aspartate, glutamate, and creatine levels, which are probably related to the impaired electron transport chain in mitochondria; high serine/glycine metabolism and so one-carbon metabolism; low glycerophosphocholine-phosphocholine ratio in membrane metabolism, which is associated with tumor aggressiveness; and finally negative MGMT methylation status.

Conclusions: It seems imperative to identify this subgroup of high-grade gliomas with low FDG avidity, which is especially aggressive. Their identification could be important for early detection for a possible personalized treatment, such as antifolate treatment.
\end{abstract}

Key Words: FDG PET, glioma, metabolomics,

HRMAS-NMR spectroscopy

(Clin Nucl Med 2019;44: 936-942)

$\mathrm{F}$ DG is the first and indisputably the most important radiotracer for imaging tumor metabolism and is used in clinical practice for tumor malignancy grading, staging, and therapeutic response evaluation in many cancers. FDG uptake reflects the increase in aerobic glycolysis with lactic fermentation used for energy production

Received for publication April 30, 2019; revision accepted July 4, 2019.

From the *Service de Biophysique et Médecine Nucléaire, Hôpitaux Universitaires de Strasbourg: †MNMS-Platform, Hôpital de Hautepierre, Hôpitaux Universitaires de Strasbourg; †ICube, Université de Strasbourg/CNRS, UMR 7357; §Service d'Anatomie Pathologique, Hôpital de Hautepierre, Hôpitaux Universitaires de Strasbourg, Strasbourg, France; ||Computer Engineering Department, Bilkent University, Ankara, Turkey; $\uparrow$ Computational Biology Department, School of Computer Science, Carnegie Mellon University, Pittsburgh, PA; and **Service de Neurochirurgie, Hôpital de Hautepierre, Hôpitaux Universitaires de Strasbourg, Strasbourg, France.

Ethics approval and consent to participate: The Ethics Committee of Strasbourg approved the study (no. 2003-100, September 12, 2003 and no. 2013-37, December 11, 2013). Written informed consent was obtained from all patients included.

Informed consent: Informed consent was obtained from all individual participants included in the study. A written informed consent was obtained from all the patients included.

Conflicts of interest and sources of funding: none declared.

Correspondence to: Caroline Bund, MD, MSc, Service de Biophysique et de Médecine Nucléaire, Hôpitaux Universitaires de Strasbourg, Hôpital de Hautepierre, 1, Avenue Molière, 67098 Strasbourg Cedex 09, France. E-mail: Caroline.BUND@chru-strasbourg.fr.

Copyright (C) 2019 Wolters Kluwer Health, Inc. All rights reserved.

ISSN: 0363-9762/19/4412-0936

DOI: $10.1097 /$ RLU.0000000000002765
(Warburg phenomenon) and/or the anabolic reactions (glucose is a major donor), both involved in tumor growth.

In gliomas, FDG PET is also a useful tool for the evaluation of the degree of malignancy. It is commonly accepted that highgrade gliomas are characterized by increased FDG uptake and that low-grade gliomas reduced FDG uptake. This property has been used to diagnose malignant transformation of low-grade gliomas and for the management of high-grade gliomas to guide biopsies, to assess posttreatment response, and to detect early tumor relapse compared with radionecrosis. $^{1-6}$

Few studies have focused on high-grade gliomas with reduced glucose avidity. ${ }^{3,7,8}$ The aim of this study was to compare the tumor metabolomics using high-resolution magic-angle spinning (HRMAS) nuclear magnetic resonance (NMR) spectroscopy in 2 populations of high-grade gliomas presenting high or low FDG uptake. We also compared the tumor metabolomics of high-grade gliomas with low FDG uptake and low-grade gliomas presenting low FDG uptake.

\section{MATERIALS AND METHODS}

\section{Patient Population}

The high-grade glioma group consisted of 12 patients, 8 patients with a high-grade astrocytoma ( 7 glioblastomas and 1 grade III astrocytoma, World Health Organization [WHO] 2016) and 4 patients with a high-grade oligodendroglioma (grade III, WHO 2016). The patient characteristics are listed in Table 1. For 8 high-grade astrocytomas, 4 patients presented high FDG uptake (normalized SUV [nSUV], $\geq 1.84$ ) and 4 patients presented low FDG uptake (nSUV, $\leq 0.85$ ) despite the same histologic and morphologic aspects on MRI, that is, intense contrast enhancement, central necrosis, and expanded perilesional edema (Fig. 1). Of the 4 patients with highgrade oligodendrogliomas, 3 presented high FDG uptake (nSUV, $\geq 1.43$ ) and 1 patient presented low FDG uptake (nSUV, 0.66) despite the same histologic, MRI, and F-DOPA PET aspects (Fig. 2).

The low-grade glioma group consisted of 6 patients (1 with grade II astrocytoma and 5 with grade II oligodendroglioma, WHO 2016). In this group, the mean age was 34.2 years (range, 19-46 years) with 5 women and 1 man. Mean overall survival was 79.5 months (range, 38.5-91.9 months). All patients are still alive. In all cases, we observed the same morphologic aspects on MRI (no contrast enhancement, no necrosis, and no significant perilesional edema) and the same metabolic imaging aspects, that is, low FDG uptake (mean nSUV, 0.48; range, 0.41-0.55; standard deviation, 0.06) and moderate F-DOPA uptake in PET (Figs. 1, 2).

All patients fulfilled the following criteria: (1) histopathological confirmation of glioma grading after rereading; (2) presence of FDG PET imaging before surgery; and (3) presence of tumor tissue samples in the Strasbourg University Hospitals' Tumor Bio-bank for HRMAS NMR spectroscopy analysis.

\section{FDG PET Imaging}

PET examinations were performed on a GE Discovery ST (GE Medical System, Milwaukee, WI) until May 2013 and on a Siemens mCT128 Biograph (Siemens Medical Solutions, Erlangen, 


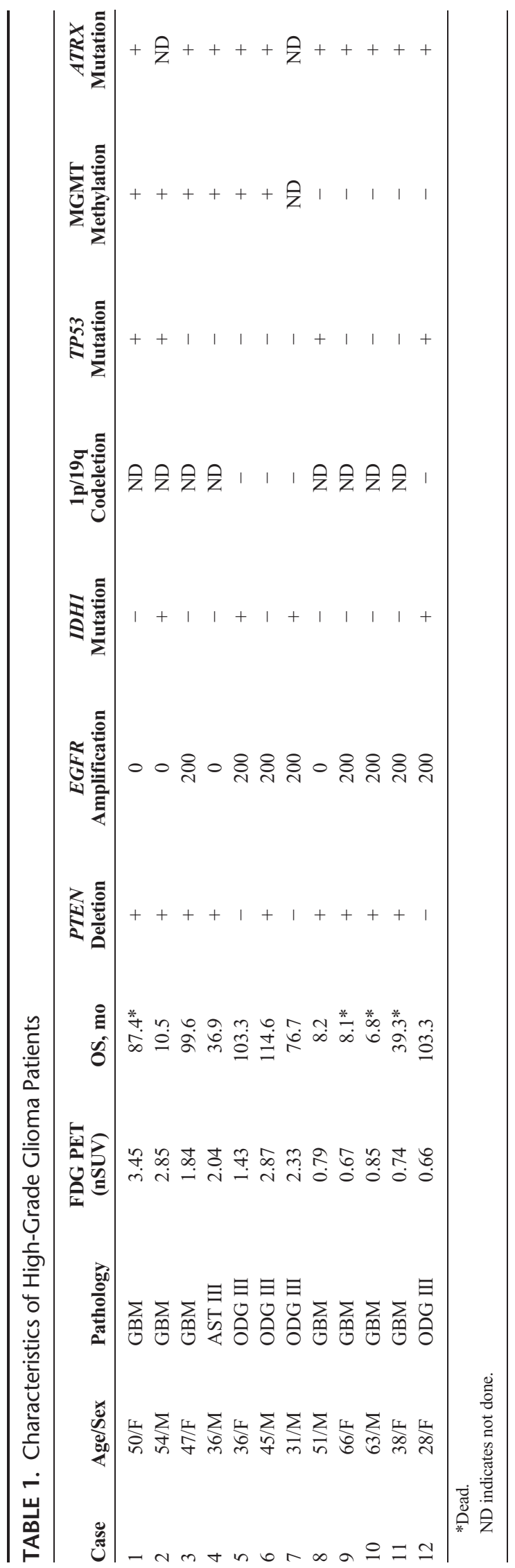

Germany) after May 2013. ${ }^{18}$ F-FDG was injected intravenously at $2.5 \mathrm{MBq} / \mathrm{kg}$ and $2 \mathrm{MBq} / \mathrm{kg}$, respectively, after at least 6 hours of fasting (with the exception of ad libitum water intake) and capillary glycemia lower than $6.6 \mathrm{mmol} / \mathrm{L}$. Image acquisition was initiated 30 minutes and 4 hours after ${ }^{18} \mathrm{~F}$-FDG injection, including low-dose noncontrast transmission CT scan followed by a 3-dimensional PET scan with an acquisition time of 15 minutes. PET data were reconstructed with and without CT-based attenuation correction using a common iterative algorithm (ordered subset expectation maximization, 2 iterations, 21 subsets, matrix $128 \times 128$ ).

For semiquantitative analysis of FDG uptake, we used the $\mathrm{SUV}_{\max }$ per focus defined as: $\mathrm{SUV}=$ [maximum value of radioactivity concentration $(\mathrm{kBq} / \mathrm{mL})] /[$ injected dose $(\mathrm{MBq}) /$ patient weight $(\mathrm{kg})]$. Normalized SUV was calculated as the ratio of maximum tumor FDG uptake obtained using the volume of interest covering the entire tumor to maximum cerebellum FDG uptake. An nSUV value equal to or greater than 1 is considered hypermetabolic; less than 1 is considered hypometabolic.

\section{HRMAS NMR Analysis}

\section{Sample Preparation}

Tissue specimens were collected with minimum ischemic delays after resection (average time $2 \pm 1$ minute) and snap-frozen in liquid nitrogen before being stored at $-80^{\circ} \mathrm{C}$. All tissue samples exhibited a viable tumor/necrosis ratio and were quantitatively and qualitatively adequate to perform satisfactory HRMAS analysis. Each brain biopsy sample was prepared at $-20^{\circ} \mathrm{C}$ by introducing a 15- to 18-mg biopsy into a disposable 30- $\mu \mathrm{L} \mathrm{KelF} \mathrm{insert.} \mathrm{To} \mathrm{provide}$ a lock frequency for the NMR spectrometer, $10 \mu \mathrm{L}$ of $\mathrm{D}_{2} \mathrm{O}$ was also added to the insert.

\section{HRMAS NMR Data Acquisition}

All HRMAS NMR spectra were acquired on a Bruker (Karlsruhe, Germany) Avance III 500 spectrometer operating at a proton frequency of $500.13 \mathrm{MHz}$ and equipped with a 4-mm tripleresonance gradient HRMAS probe $\left({ }^{1} \mathrm{H},{ }^{3} \mathrm{C}\right.$, and $\left.{ }^{31} \mathrm{P}\right)$. The temperature was maintained at $4^{\circ} \mathrm{C}$ throughout the acquisition time to reduce the effects of tissue degradation during the spectrum acquisition. A 1-dimensional proton spectrum using a Carr-Purcell-Meiboom-Gill (CPMG) pulse sequence was acquired with a $285-\mu$ s interpulse delay and a 10-minute acquisition time for each tissue sample. The number of loops was set at 328, giving the CPMG pulse train a total length of 93 milliseconds. The chemical shift was calibrated to the peak of the methyl proton of L-lactate at $1.33 \mathrm{ppm}$. To confirm resonance assignments in a few representative samples, 2-dimensional heteronuclear experiments $\left({ }^{1} \mathrm{H}-{ }^{13} \mathrm{C}\right)$ were also recorded immediately after ending the 1-dimensional spectra acquisition.

\section{HRMAS NMR Data Processing}

Metabolite assignation and quantification was done with Chenomx software (Edmonton, Alberta, Canada), using a database of NMR spectra of 76 metabolites acquired in our laboratory under the same CPMG pulse sequence as the tissue samples. ${ }^{9}$ We could detect and quantify 42 metabolites in glioma samples: acetate, adenosine, alanine, allocystathionine, arginine, ascorbate, asparagine, aspartate, betaine, choline, creatine, ethanolamine, fumarate, gamma-aminobutyric acid (GABA), glycine, glucose, glutamate, glutamine, glutathione (GSH), glycerol, glycerophosphocholine (GPC), hydroxybutyrate, 2-hydroxyglutarate ( $2 \mathrm{HG})$, hypotaurine, isoleucine, lactate, lysine, methionine, myoinositol, $N$-acetyl-aspartate (NAA), N-acetyl-lysine, ornithine, phenylalanine, phosphocholine (PC), proline, serine, scylloinositol, succinate, taurine, threonine, tyrosine, and valine. The results are expressed in nmol. $\mathrm{mg}^{-1}$ of tissue. We 

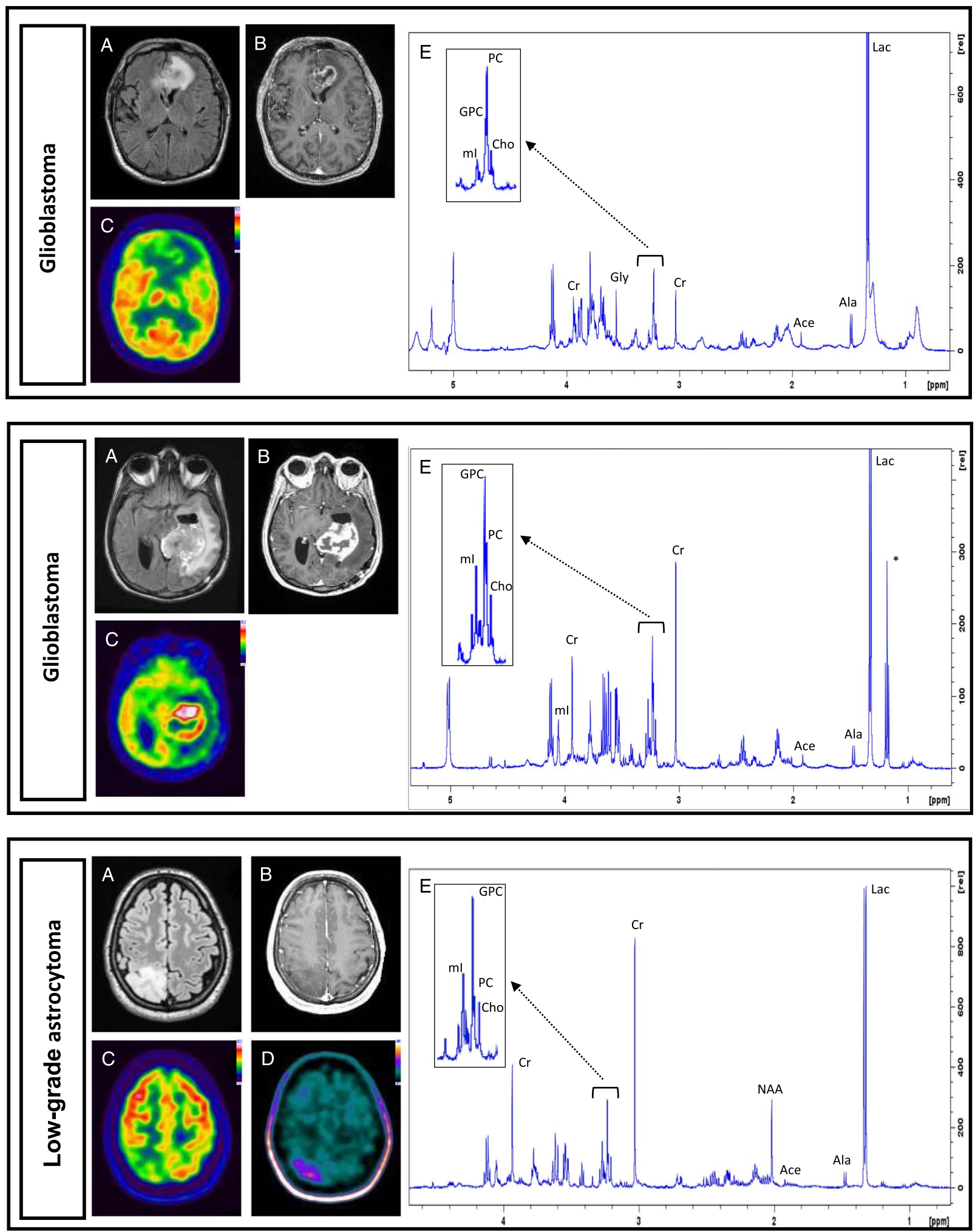

FIGURE 1. Three representative cases for astrocytomas: 2 different metabolic patterns of glioblastomas (first with low FDG uptake and second with high FDG uptake) compared with low-grade astrocytoma (third case). For each one, we showed matched transverse slices of FLAIR (A) and Gd-enhanced T1-weighted (B) MRI, FDG PET (C), FDOPA PET (D), and HRMAS NMR spectra (E). HRMAS NMR spectra demonstrates clearly inverted GPC/PC ratio, increased glycine (Gly), decreased creatine $(\mathrm{Cr})$, and decreased myoinositol (ml) levels in glioblastoma with low FDG uptake (first case). Levels of acetate (Ace), alanine (Ala), lactate (Lac), and choline (Cho) remain similar. The asterisk shows ethanol contamination. 

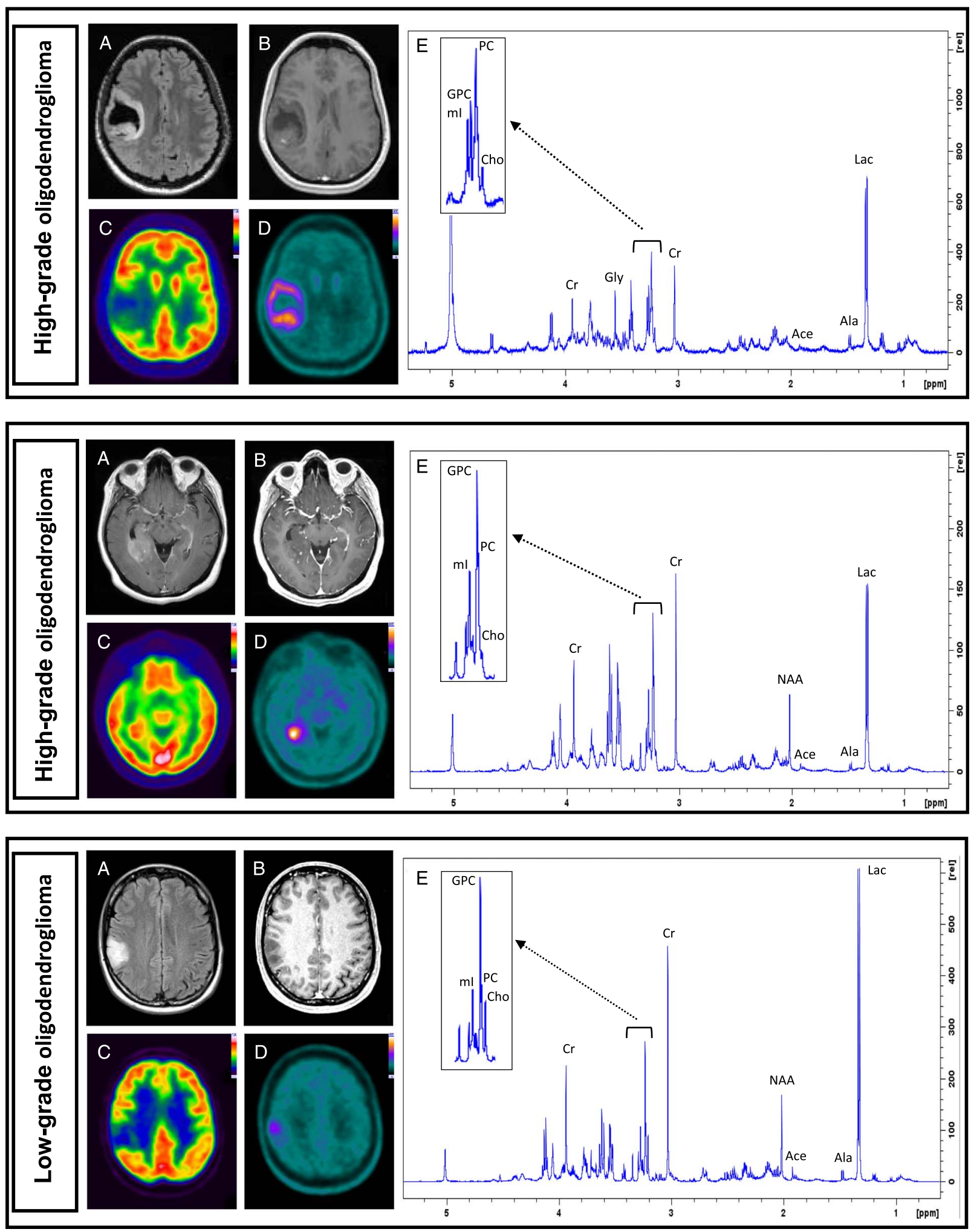

FIGURE 2. Three representative cases for oligodendrogliomas: 2 different metabolic patterns of high-grade oligodendrogliomas (first with low FDG uptake and second with high FDG uptake) compared with low-grade oligodendroglioma (third case). For each one, we showed matched transverse slices of FLAIR (A) and Gd-enhanced T1-weighted (B) MRI, FDG PET (C), FDOPA PET (D), and HRMAS NMR spectra (E). HRMAS NMR spectra demonstrates, as in glioblastoma cases, inverted GPC/PC ratio, increased glycine (Gly), and decreased creatine ( $\mathrm{Cr}$ ) levels in high-grade oligodendroglioma with low FDG uptake (first case). Levels of acetate (Ace), alanine (Ala), lactate (Lac), myoinositol (ml), and choline (Cho) remain similar. 
used also total choline (choline + GPC + PC) as additional parameters in network analysis.

\section{Network Analysis}

The algorithm to determine the expected metabolite level alterations (ADEMA) network analyses using mutual information were applied to the metabolite quantification values. ${ }^{10}$ ADEMA includes information on the metabolic pathway in a unidirectional or bidirectional manner. The network was constructed using the Kyoto Encyclopedia of Genes and Genomes ${ }^{11,12}$ and Salway's work. ${ }^{13}$ Using the metabolic network topology, the ADEMA algorithm evaluates the change in groups of metabolites between concentration data from 2 experimental groups instead of analyzing metabolite concentrations one by one. Based on mutual information, the algorithm determines whether some metabolites are biomarkers when considered together, and it can predict the direction of the expected change per metabolite depending on the metabolic network topology considered. Various groups of metabolites related to the metabolic pathways involved were compared):

- Taurine, hypotaurine, aspartate, methionine, allocystathionine, serine

- Aspartate, asparagine, acetate, threonine, NAA

- Aspartate, lysine, $N$-acetyl-lysine

- Acetate, threonine, allocystathionine, methionine

- Glucose, acetate, hydroxybutyrate

- Aspartate, threonine, isoleucine

- Glucose, glycine, serine

- Glucose, glycerol, phenylalanine, tyrosine

- Glucose, valine, isoleucine

- Glucose, lactate

- Valine, lactate, alanine

- Glucose, myoinositol, ascorbate, GSH, glycine, glutamate

- Myoinositol, scylloinositol

- Glutamate, GABA, proline

- Aspartate, adenosine, succinate, fumarate, $2 \mathrm{HG}$

- Glutamate, glutamine, glycine, $2 \mathrm{HG}$

- Glutamate, arginine, glycine, creatine, ornithine

- Aspartate, arginine, ornithine

- Ethanolamine, choline, GPC, PC, total choline

- Choline, betaine, glycine

\section{Histopathology}

After NMR HRMAS analysis, the inserts were cut, and for half the content of each sample, the percentage of tumor cells in the total sample of cells with regard to the total surface were calculated based on frozen hematoxylin and eosin-stained sections.

\section{RESULTS}

Table 2 summarizes the network analysis results obtained on metabolite concentration comparisons between on one hand highgrade gliomas with low and high FDG uptake, and on the other hand high-grade gliomas with low-grade gliomas.

\section{High-Grade Gliomas}

The HRMAS NMR spectra of high-grade glioma patients with low or high FDG uptake are clearly different (Figs. 1, 2). The ADEMA network analysis shows that the low FDG update was associated with an elevated concentration for taurine, hypotaurine, valine, isoleucine, GSH, serine, glycine, PC, lysine, hydroxybutyrate, phenylalanine, and tyrosine, and a low concentration for allocystathionine, glucose, myoinositol, scylloinositol, ascorbate, glutamate, $2 \mathrm{HG}$, aspartate, creatine, NAA, acetate, ethanolamine, GPC, total choline, $N$-acetyl-lysine, GABA, proline, adenosine, glycerol, and betaine (Fig. 3, Table 2).

\section{Comparison With Low-Grade Gliomas}

Compared with low-grade gliomas, high-grade gliomas with low FDG uptake were associated with an elevated concentration for methionine, glucose, alanine, valine, isoleucine, GSH, serine, glycine, fumarate, asparagine, PC, $N$-acetyl-lysine, hydroxybutyrate, ornithine, phenylalanine, and tyrosine, and a low concentration for allocystathionine, myoinositol, scylloinositol, ascorbate, glutamate, succinate, $2 \mathrm{HG}$, creatine, aspartate, NAA, arginine, acetate, ethanolamine, GPC, total choline, GABA, adenosine, glycerol, and betaine (Table 2).

Compared with low-grade gliomas, high-grade gliomas with high FDG uptake were associated with an elevated concentration for methionine, glucose, alanine, valine, isoleucine, fumarate, acetate, asparagine, choline, $\mathrm{PC}$, and ornithine, and a low concentration for allocystathionine, myoinositol, scylloinositol, GSH, succinate, 2HG, NAA, creatine, arginine, GPC, GABA, and adenosine (Table 2).

\section{DISCUSSION}

The results presented in this study demonstrate that the highgrade gliomas with low FDG avidity are more aggressive than the high-grade gliomas with high FDG avidity. Whether originating from astrocytes or oligodendrocytes, these gliomas form a subgroup of high-grade gliomas with common characteristics.

First, low aspartate, glutamate, and creatine levels are probably related to the impaired electron transport chain in mitochondria and considered as a predictive metabolic marker of the degree of hypoxia. ${ }^{14}$ Even if there are no significant differences in alanine and lactate levels, high levels of many amino acids produced by anaerobic pathways (valine, isoleucine, lysine, etc) and a high GSH level showing cellular oxidative stress support this idea.

Second, high serine/glycine metabolism is probably related to overexpression of serine hydroxymethyltransferase described in many cancers and gliomas and is associated with a poor prognosis. ${ }^{15}$ In anabolic pathways, the serine/glycine pathway represents a crucial turning point in glucose conversion. ${ }^{16}$ Imported serine and serine derived from a branch of glycolysis can be converted to glycine, which in turn provides carbon units for one-carbon metabolism. ${ }^{17,18}$ The synthesis of proteins, lipids, nucleic acids, and other cofactors requires one-carbon metabolism, which is a complex metabolite network that is based on the chemical reactions of folate compounds. The one-carbon unit proceeds in a cyclical pathway from which it is transferred to other metabolic pathways. The importance of this metabolic pathway is underlined by the fact that antifolate chemotherapy is currently widely used in cancer treatment and has been since its discovery more than 50 years ago. ${ }^{18}$

Third, there is a decreased GPC/PC ratio in membrane metabolism, which is associated with tumor aggressiveness, ${ }_{19,20}$ knowing that increased choline kinase activity enhances the PC level and increased glycerophosphodiesterase activity decreases the GPC level. ${ }^{21-23}$

Finally, the MGMT methylation status, which predicts the beneficial effect of temozolomide treatment in glioblastoma, ${ }^{24,25}$ is systematically absent in low-FDG-uptake cases (Table 1).

\section{CONCLUSIONS}

This study demonstrates that there exists a subgroup of high-grade gliomas especially aggressive that can be identified using FDG PET, which shows low FDG avidity, and in vivo MR spectroscopy, which detects low creatine level. ${ }^{26}$ Their identification could be important for early detection for a possible personalized treatment. 
TABLE 2. Summary of ADEMA Network Analysis Conducted for Specified Groups Comparison Based on Metabolite Concentration Obtained by HRMAS NMR Spectroscopy

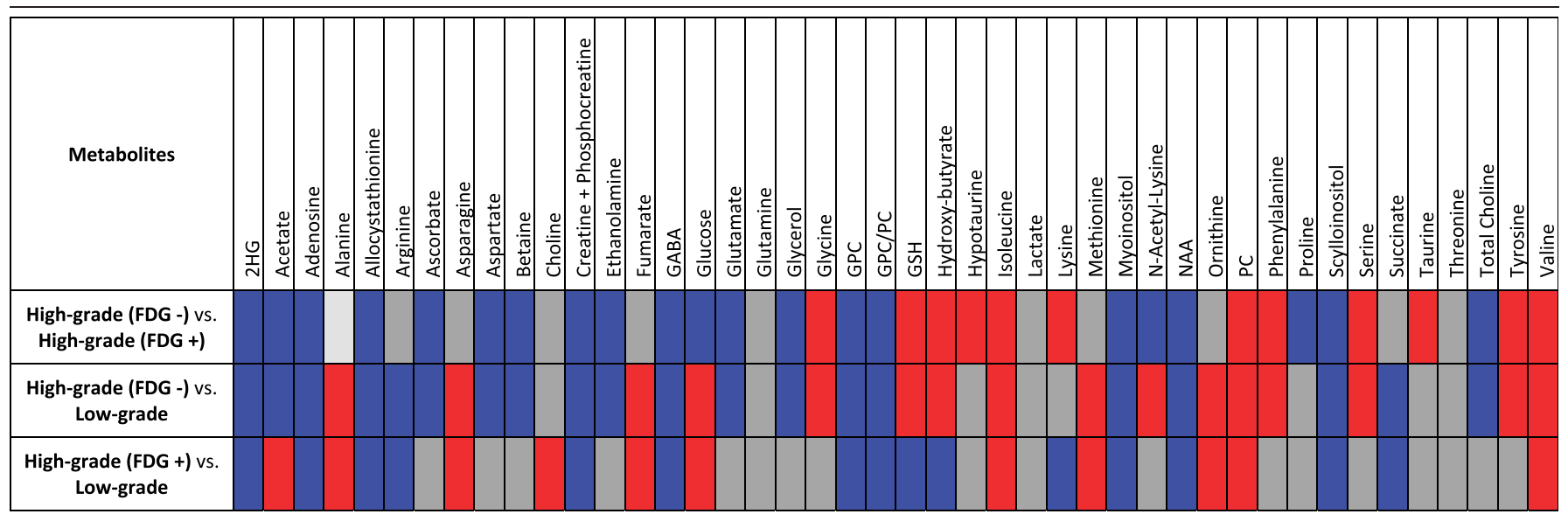

FDG - and FDG + show respectively low and high FDG uptake.

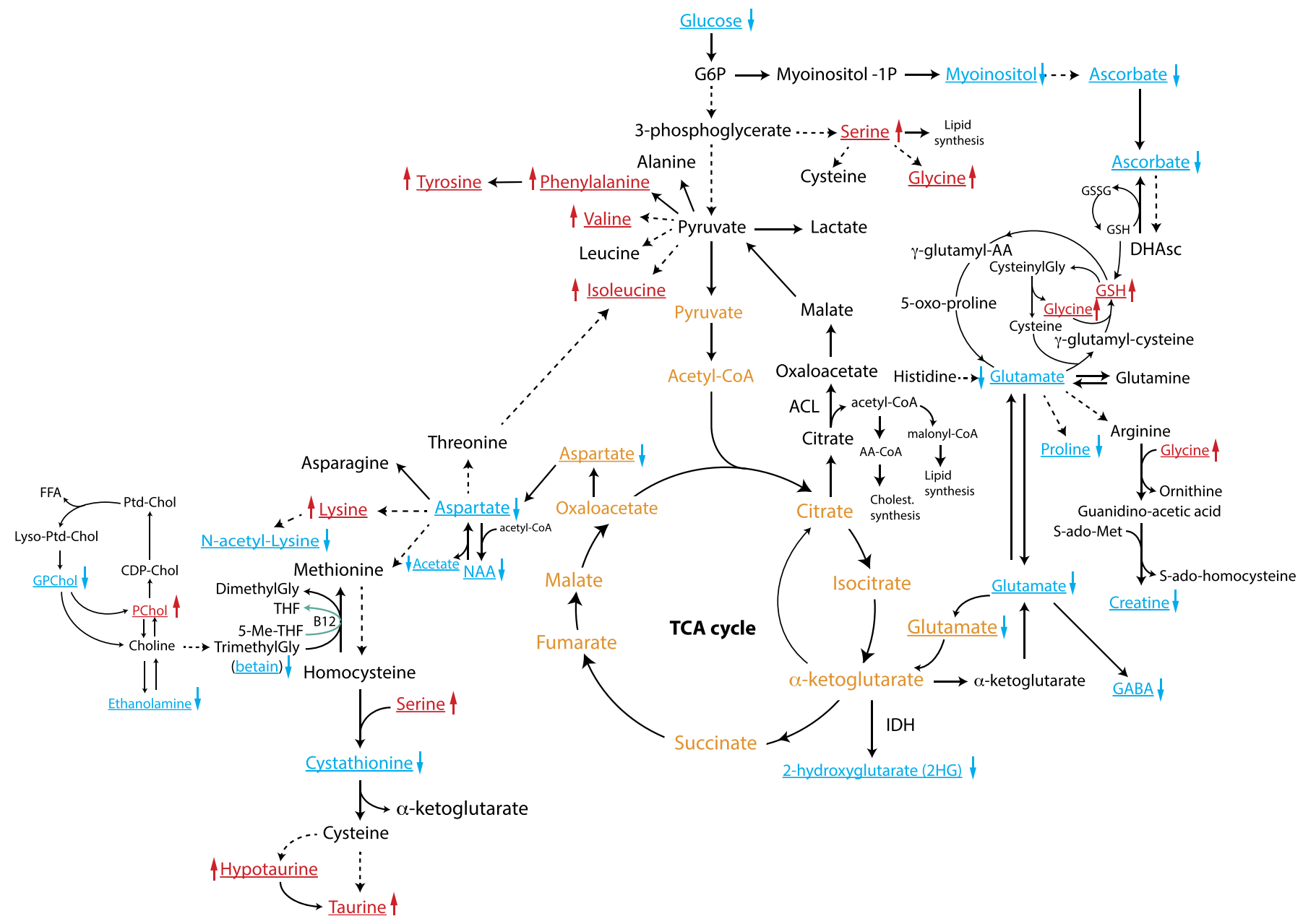

FIGURE 3. Results of network analysis between high-grade gliomas with low FDG uptake compared with high-grade gliomas with high FDG uptake. The metabolites underlined and written in red and blue with an arrow for each one indicate the metabolites that are predicted to increase and decrease in high-grade gliomas with low FDG uptake. The metabolites in orange are present in the mitochondria. 


\section{ACKNOWLEDGMENTS}

The laboratory staff of Strasbourg University Hospitals' Tumor Bio-bank (Centre de Ressources Biologiques) is gratefully acknowledged for their technical assistance.

\section{REFERENCES}

1. Chen W, Silverman DH. Advances in evaluation of primary brain tumors. Semin Nucl Med. 2008;38:240-250.

2. De Witte O, Lefranc F, Levivier M, et al. FDG-PET as a prognostic factor in high-grade astrocytoma. J Neurooncol. 2000;49:157-163.

3. Padma MV, Said S, Jacobs M, et al. Prediction of pathology and survival by FDG PET in gliomas. $J$ Neurooncol. 2003;64:227-237.

4. Hanson MW, Glantz MJ, Hoffman JM, et al. FDG-PET in the selection of brain lesions for biopsy. J Comput Assist Tomogr. 1991;15:796-801.

5. Levivier M, Goldman S, Bidaut LM, et al. Positron emission tomographyguided stereotactic brain biopsy. Neurosurgery. 1992;31:792-797; discussion 797.

6. Dankbaar JW, Snijders TJ, Robe PA, et al. The use of (18)F-FDG PET to differentiate progressive disease from treatment induced necrosis in high grade glioma. J Neurooncol. 2015;125:167-175.

7. Davis WK, Boyko OB, Hoffman JM, et al. $\left[{ }^{18} \mathrm{~F}\right] 2$-fluoro-2-deoxyglucosepositron emission tomography correlation of gadolinium-enhanced MR imaging of central nervous system neoplasia. AJNR Am J Neuroradiol. 1993; 14:515-523.

8. Atkinson M, Juhász C, Shah J, et al. Paradoxical imaging findings in cerebral gliomas. J Neurol Sci. 2008;269:180-183.

9. Ruhland E, Bund C, Outilaft H, et al. A metabolic database for biomedical studies of biopsy specimens by high-resolution magic angle spinning nuclear MR: a qualitative and quantitative tool. Magn Reson Med. 2019.

10. Cicek AE, Bederman I, Henderson L, et al. ADEMA: an algorithm to determine expected metabolite level alterations using mutual information. PLoS Comput Biol. 2013;9:e1002859.

11. Kanehisa M, Goto S. KEGG: Kyoto Encyclopedia of Genes and Genomes. Nucleic Acids Res. 2000;28:27-30.

12. Kanehisa M, Goto S, Sato Y, et al. Data, information, knowledge and principle: back to metabolism in KEGG. Nucleic Acids Res. 2014;42:D199-D205.
13. Salway JG. Metabolism at a Glance. 4th ed. Hoboken, New Jersey: Wiley-Blackwell Publishing; 2017.

14. Garcia-Bermudez J, Baudrier L, La K, et al. Aspartate is a limiting metabolite for cancer cell proliferation under hypoxia and in tumours. Nat Cell Biol. 2018;20:775-781.

15. Wang DW, Wu L, Cao Y, et al. A novel mechanism of mTORC1-mediated serine/glycine metabolism in osteosarcoma development. Cell Signal. 2017;29:107-114.

16. Kalhan SC, Hanson RW. Resurgence of serine: an often neglected but indispensable amino Acid. J Biol Chem. 2012;287:19786-19791.

17. Geck RC, Toker A. Nonessential amino acid metabolism in breast cancer Adv Biol Regul. 2016;62:11-17.

18. Amelio I, Cutruzzolá F, Antonov A, et al. Serine and glycine metabolism in cancer. Trends Biochem Sci. 2014;39:191-198.

19. Dali-Youcef N, Froelich S, Moussallieh FM, et al. Gene expression mapping of histone deacetylases and co-factors, and correlation with survival time and 1H-HRMAS metabolomic profile in human gliomas. Sci Rep. 2015;5:9087.

20. Bund C, Guergova-Kuras M, Cicek AE, et al. An integrated genomic and metabolomic approach for defining survival time in adult oligodendrogliomas patients. Metabolomics. 2019.

21. Cuadrado A, Carnero A, Dolfi F, et al. Phosphorylcholine: a novel second messenger essential for mitogenic activity of growth factors. Oncogene. 1993;8:2959-2968.

22. Glunde K, Shah T, Winnard PT, et al. Hypoxia regulates choline kinase expression through hypoxia-inducible factor-1 alpha signaling in a human prostate cancer model. Cancer Res. 2008;68:172-180.

23. Stewart JD, Marchan R, Lesjak MS, et al. Choline-releasing glycerophosphodiesterase EDI3 drives tumor cell migration and metastasis. Proc Natl Acad Sci U S A. 2012;109:8155-8160.

24. Wick W, Platten M, Meisner C, et al. Temozolomide chemotherapy alone versus radiotherapy alone for malignant astrocytoma in the elderly: the NOA-08 randomised, phase 3 trial. Lancet Oncol. 2012;13:707-715.

25. Malmström A, Grønberg BH, Marosi C, et al. Temozolomide versus standard 6-week radiotherapy versus hypofractionated radiotherapy in patient older than 60 years with glioblastoma: the Nordic randomised, phase 3 trial Lancet Oncol. 2012;13:916-926.

26. Ben Sellem D, Heymann S, Imperiale A, et al. Importance of metabolic imaging in monitoring glioblastomas. Médecine Nucléaire. 2008;32:205. 